\title{
Modeling of Compact, Broad-stopband Lowpass Filter using Modified Stepped Impedance Hairpin Resonators
}

\author{
Sayed Ashraf Mamun and Abdul Matin, Member, IEEE
}

\begin{abstract}
A compact elliptical function lowpass filter using modified stepped impedance hairpin resonators is proposed. The filter is analyzed from the equivalent lumped-element model using transmission line theory. The model is evaluated with EM simulation with good agreement. For single lowpass filter, the return loss is better than $13 \mathrm{~dB}$ from $D C$ to $1.5 \mathrm{GHz}$ where the insertion loss is less than $0.5 \mathrm{~dB}$ with considerable pass band ripple, $\pm 0.3 \mathrm{~dB}$. The rejection is greater than $-15 \mathrm{~dB}$ from $2.0 \mathrm{GHz}$ to $10 \mathrm{GHz}$. By cascading proposed lowpass filters with different length and width, more rejection in stopband, low insertion loss, broad stopband can be obtained to design an efficient filter for many wireless communication systems.
\end{abstract}

Index Terms - Low pass filter, micro strip, hairpin resonator, broad stopand.

\section{INTRODUCTION}

Microstrip hairpin resonator has been drawing much attention to microwave filters designing. From half-wave length to the stepped impedance resonator, a broad stop band and compact size has been emerging in recent research [1]-[4]. Microstrip elliptical filter has more advantage than Chebyshev and Butterworth filters, i.e, high performance, low cost and easy fabrication [5-6]. However, L. H. Hsieh etal. has modelled the microstrip stepped impedance hairpin resonators with transmission line theory [7]. It has broad stopband and compact in size. An improved design has been proposed by M. H. Yang with modified stepped impedance hairpin resonator [8]. But it has not been yet modeled in circuit elements.

In this paper, a new microstrip lowpass filter using stepped impedance hairpin resonator with a pair of couple triangular stepped impedance resonators inside is modeled. The couple stepped impedance resonators are used as a capacitor to have sharp cutoff and create transmission zero for a broad stopband. At the same time it has been optimized by EM simulation. For the simulation Agilent Advanced Design System (ADS) version:2011.01 is used.

\section{EQuivalent Circuit FOR MOdifiEd STEPPED IMPEDANCE HAIRPIN}

Fig-1 shows the layout of modified stepped impedance hairpin resonator. It has three parts- series line (fig-2(a)),

Manuscript received July 19, 2011; revised August 25, 2011.

The authors are with the Department of Electrical and Electronic Engineering, Bangladesh University of Engineering and Technology, Dhaka-1000, Bangladesh (Phone: +8801911348014; e-mail: mitulbabu@yahoo.com). parallel couple line (fig-2(b)) and the modified triangular couple line (fig-2(c)). First, the series line has a length of $l_{s}$ $=2 l_{3}+l_{2}$ and width of $w_{2}$. As the length, $l_{s}$ is larger than the width, $w_{2}$, it shows good inductance. Then the parallel couple line has large width, $w_{3}$, length, $l_{4}$ and gap between parallel plates, $g$. The modified triangular couple line has a length, $l_{6}$, width, $w_{c}{ }^{\prime}=l_{2}-2 . w_{4}$ and a gap between the triangular plates, $x$. If $Z_{s}$ is the characteristic impedance of the single transmission line $l_{s}, Z_{o}$ and $Z_{e}$ are the odd and even impedance for the parallel couple line, we took $Z_{s}>\sqrt{Z_{o} Z_{e}}$ to reduce the size of conventional hairpin resonator [3].

ABCD parameter of Pi-model of single transmission line which has a length of $l_{s}$ is shown here below,

$$
\left(\begin{array}{ll}
A & B \\
C & D
\end{array}\right)=\left(\begin{array}{cc}
\cos \left(\beta_{s} l_{s}\right) & j Z_{s} \sin \left(\beta_{s} l_{s}\right) \\
j Y_{s} \sin \left(\beta_{s} l_{s}\right) & \cos \left(\beta_{s} l_{s}\right)
\end{array}\right)
$$

where, $\beta_{s}$ and $Y_{s}=\frac{1}{Z_{s}}$, are the phase constant and characteristics impedance. The ABCD parameter of the equivalent L-C pi-network (shown in fig-2(b)),

$$
\left(\begin{array}{ll}
A & B \\
C & D
\end{array}\right)=\left(\begin{array}{cc}
1+Z_{L} Y_{C} & Z_{L} \\
Y_{C}\left(2+Z_{L} Y_{C}\right) & 1+Z_{L} Y_{C}
\end{array}\right)
$$

where, $Z_{L}=j \omega L_{s}$ and $Y_{C}=j \omega C_{s}$.

Computing equation (1) and (2),

$$
L_{s}=\frac{Z_{s} \sin \left(\beta_{s} l_{s}\right)}{\omega}(\mathrm{H})
$$

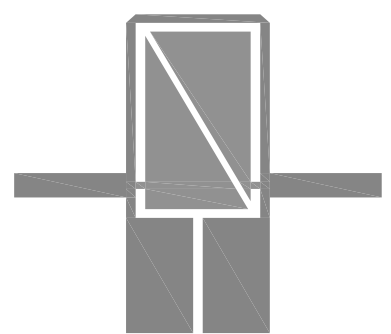

Fig. 1. Stepped impedance hairpin resonator.

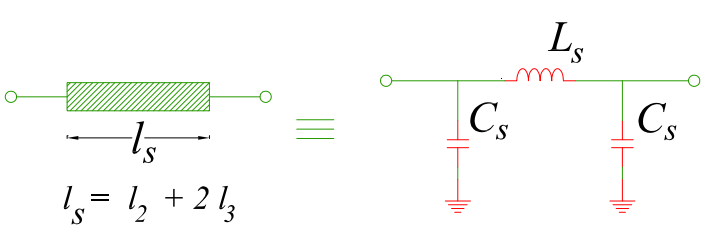

(a) 


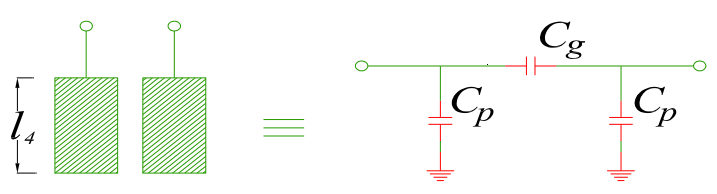

(b)
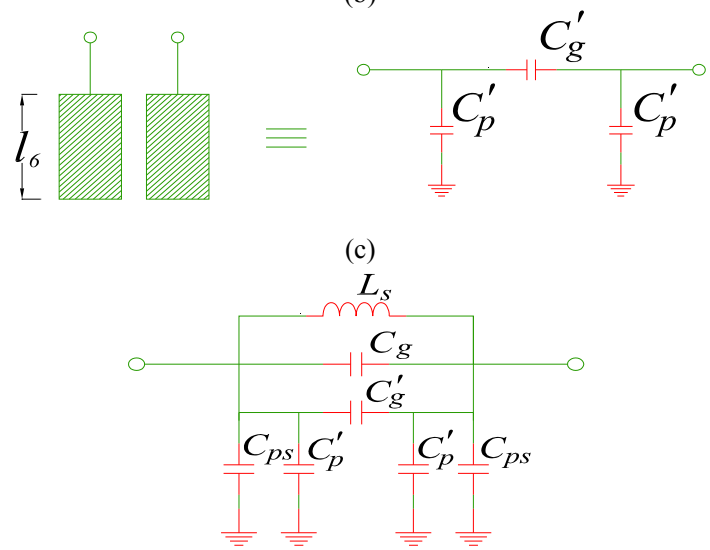

(d)

Fig. 2. Equivalent circuit of (a) single transmission line, (b) Symmetric couple lines, and (c) triangular line capacitance (d) stepped impedance hairpin resonator by combining (a), (b) and (c).

$$
C_{s}=\frac{1-\cos \left(\beta_{s} l_{s}\right)}{\omega Z_{s} \sin \left(\beta_{s} l_{s}\right)}
$$

Now the symmetric triangular couple line can be shown as an equivalent circuit (fig-2(c)). The elements of this equivalent circuit can be derived from $Z_{o}$ and $Z_{e}$. ABCD parameter for symmetric parallel couple line is shown as below [9]:

$$
\left(\begin{array}{ll}
A & B \\
C & D
\end{array}\right)=\left(\begin{array}{cc}
\frac{Z_{e} \cot \theta_{e}+Z_{o} \cot \theta_{o}}{Z_{e} \cot \theta_{e}-Z_{o} \cot \theta_{e}} & -j \frac{2 Z_{e} Z_{o} \cot \theta_{e} \cot \theta_{o}}{Z_{e} \cot \theta_{e}-Z_{o} \cot \theta_{o}} \\
j \frac{2}{Z_{e} \cot \theta_{e}-Z_{o} \cot \theta_{o}} & \frac{Z_{e} \cot \theta_{e}+Z_{o} \cot \theta_{o}}{Z_{e} \cot \theta_{e}-Z_{o} \cot \theta_{e}}
\end{array}\right)
$$

where, $\theta_{o}=\theta_{e}=\beta_{c} l_{c}$, and $\beta_{c}$ is the phase constant of the couple lines. Also the ABCD parameter of couple line equivalent circuit,

$$
\left(\begin{array}{ll}
A & B \\
C & D
\end{array}\right)=\left(\begin{array}{cc}
1+Z_{g} Y_{p} & Z_{g} \\
Y_{p}\left(2+Z_{g} Y_{p}\right) & 1+Z_{g} Y_{p}
\end{array}\right)
$$

Now comparing the two matrices in (5) and (6), the equivalent capacitance can be derived as below:

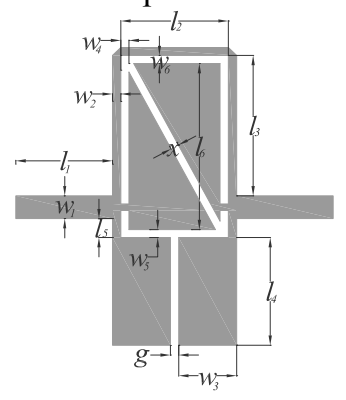

(a)

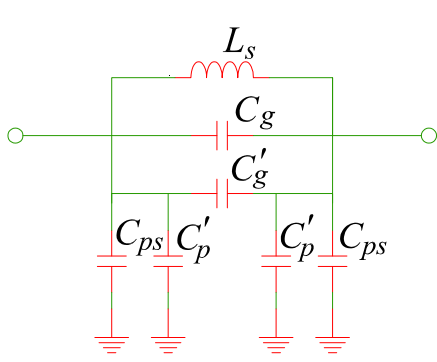

(b)
Fig. 3. Stepped impedance resonator (a) and its equivalent circuit (b) with dimension parameter.

$$
\begin{aligned}
& C_{g}=\frac{Z_{o}-Z_{e}}{2 \omega Z_{o} Z_{e} \cot \left(\beta_{c} l_{c}\right)} \\
& C_{p}=\frac{1}{\omega Z_{e} \cot \left(\beta_{c} l_{c}\right)}
\end{aligned}
$$

Now, combining these two equivalent circuit shows the final equivalent circuit where, $C_{p s}=C_{p}+C_{s}$. Here, we have neglected the junction discontinuity for simplicity.

The modified symmetric parallel couple line can be shown as an equivalent circuit (fig-2(d)) [10],

$$
\begin{aligned}
& C_{p}^{\prime}=\frac{C_{\text {even }}}{2} \\
& C_{g}^{\prime}=\frac{C_{\text {odd }}}{2}-\frac{C_{\text {even }}}{4}
\end{aligned}
$$

where, $C_{\text {odd }}$ and $C_{\text {even }}$ are the even and odd capacitance for the modified triangular coupled line.

Now, combining these three sections of equivalent circuit, the total equivalent circuit can calculated from the equation (3), (4), (7), (8), (9) and (10).

\section{COMPACt Modified ElLiptical Function Low-PASS FILTERS}

Fig-3 shows the geometry and equivalent circuit of the elliptical function low-pass filter using one modified stepped impedance hairpin resonator with feed lines, $l_{f}$. Also $L_{s}$ is the equivalent inductance of the single transmission line of the filter. $C_{g}$ is the equivalent capacitance of the couple lines and $C_{p s}$ is the sum of the capacitances of the transmission line $l_{1}$ and the couple lines. The low pass filter has been designed for a $3 \mathrm{~dB}$ cut-off frequency of $1.5 \mathrm{GHz}$ and fabricated on a $0.254 \mathrm{~mm}$ thick RT/Duroid 5880 substrate with a relative dielectric constant $\varepsilon_{r}=2.2$. The dimensions considered in this modeling are as below: $l_{1}=18 \mathrm{~mm}, l_{2}=5 \mathrm{~mm}, l_{3}=5.3 \mathrm{~mm}$, $l_{4}=6.2 \mathrm{~mm}, l_{5}=1 \mathrm{~mm}, w_{1}=0.79 \mathrm{~mm}$ for a $50 \Omega$ line, $w_{2}=0.2 \mathrm{~mm}$, and gap size $g=0.2 \mathrm{~mm}, w_{4}=w_{6}=0.2 \mathrm{~mm}, w_{5}=0.4 \mathrm{~mm}$.

The modeled filter has a $-3 \mathrm{~dB}$ passband from $\mathrm{DC}$ to $1.5 \mathrm{GHz}$ and has a better attenuation $-15 \mathrm{~dB}$ from $2.0 \mathrm{GHz}$ to $10 \mathrm{GHz}$. Its insertion loss at passband is less than $0.5 \mathrm{~dB}$ as seen in Fig-5. It has two spurious modes at $5.3 \mathrm{GHz}$ and $7 \mathrm{GHz}$. Our attention is to suppress these two spurious modes with changing its width and length as design parameter by both circuit modeling and EM simulation. The EM simulation has been carried out by Momentum (MoM simulator) of Agilent ADS. The proposed filter has a dimension of $5.4 \mathrm{~mm} \times 13.49 \mathrm{~mm}$ or $0.037 \lambda_{g} \times 0.093 \lambda_{g}$, where $\lambda_{g}$ is the guided wavelength at $1.5 \mathrm{GHz}$.

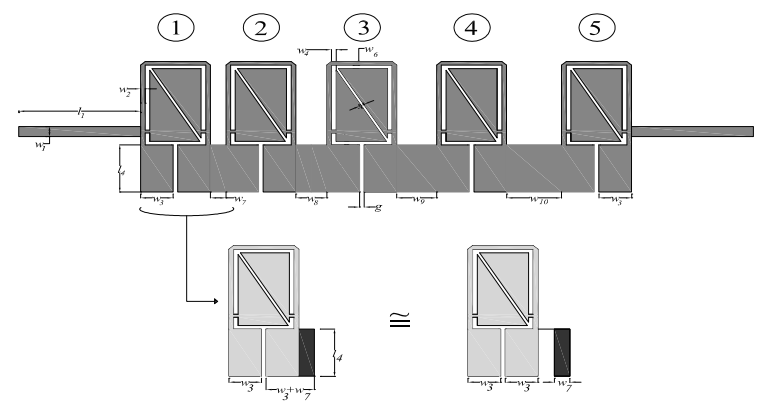

(a) 


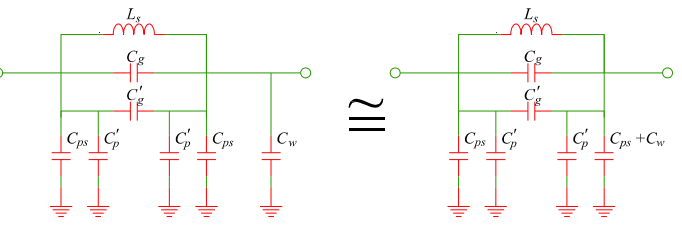

(b)

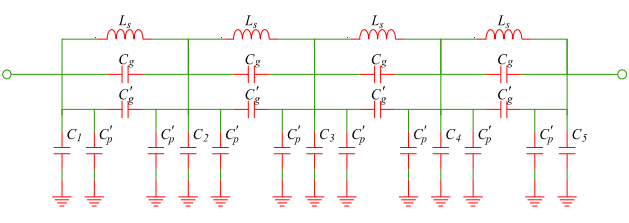

(c)

Fig. 4. (a) Layout of modified stepped impedance resonator with variation of width, (b) equivalent circuit approximation of symmetrical couple line resonator, (c) configuration of the broadband stopband lowpass filter.

\section{CASCADED Modified ElLiptical FunCTION LOW-PASS FILTERS FOR BROAD STOP-BAND}

The low pass filters using four multiples cascaded modified stepped impedance hairpin resonators is shown in Fig-4. Two resonators can be cascaded by inserting a local variable width for different resonant frequency.

Though it becomes asymmetrical resonator, it can be assumed as symmetrical for simplicity. Fig-4(a) shows the layout diagram of cascaded modified stepped impedance resonators and fig-4(b) shows the circuit diagram. If the local variable width can be thought of a shunt capacitor, $C_{s p}$ is given by,

$$
C_{s p}=\varepsilon_{0} \varepsilon_{r} w / h \quad(F / m)
$$

where, $w$ is the width of the adjacent transmission line and $h$ is the substrate height.

From fig- 6 and fig-7, the cutoff frequency form the EM simulator is $1.270 \mathrm{GHz}$ and form circuit simulation it is $1.263 \mathrm{GHz}$ with a difference of $7 \mathrm{MHz}$. This is because of negligence of small inductance created at the junction of series line and couple line.

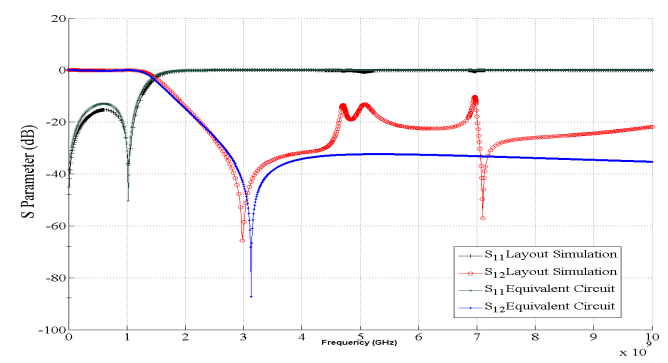

Fig. 5. EM and circuit simulated results for the lowpass filters designed for $1.5 \mathrm{GHz} 3 \mathrm{~dB}$ cut off frequency.

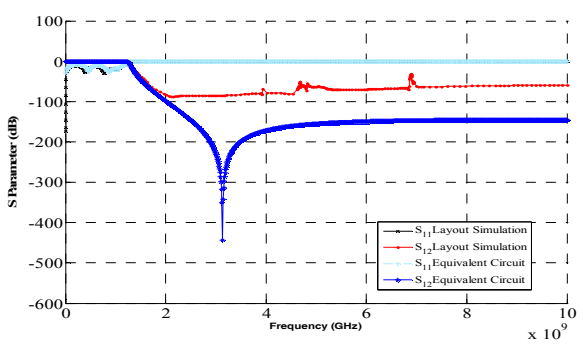

Fig. 6. Simulated results of the broad stopband lowpass filter with approximated dimensions while different width is considered.

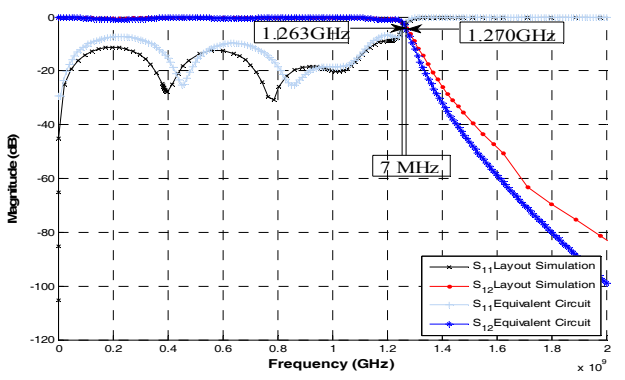

Fig. 7. Comparison of circuit model and EM simulation with approximation of dimension. Here, difference between these two cut-off frequencies is $7 \mathrm{MHz}$, return loss is lower than $-13 \mathrm{~dB}$ and passband ripple is less than $\pm 0.42 \mathrm{~dB}$

After $3 \mathrm{~dB}$ cutoff frequency, rejection region goes below $-80 \mathrm{~dB}$ in $\mathrm{EM}$ simulation and $\mathrm{a}-140 \mathrm{~dB}$ in circuit simulation. Here, circuit simulation only approximates the cutoff frequency and rejection degree in stopband. The ripple at passband is $\pm 0.4 \mathrm{~dB}$.

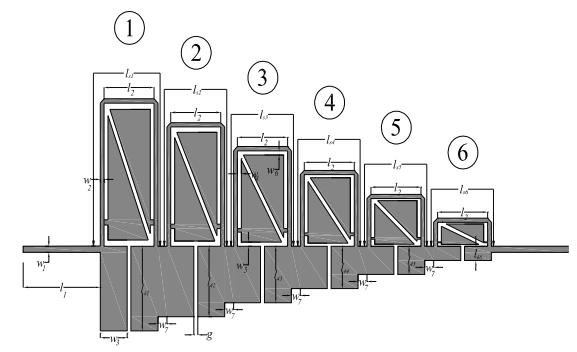

Fig. 8. Layout of modified stepped impedance resonator by cascading with variation of its length.

TABLE I: CIRCUIT ELEMENTS OF CASCADED MODIFIED STEPPED IMPEDANCE RESONATOR

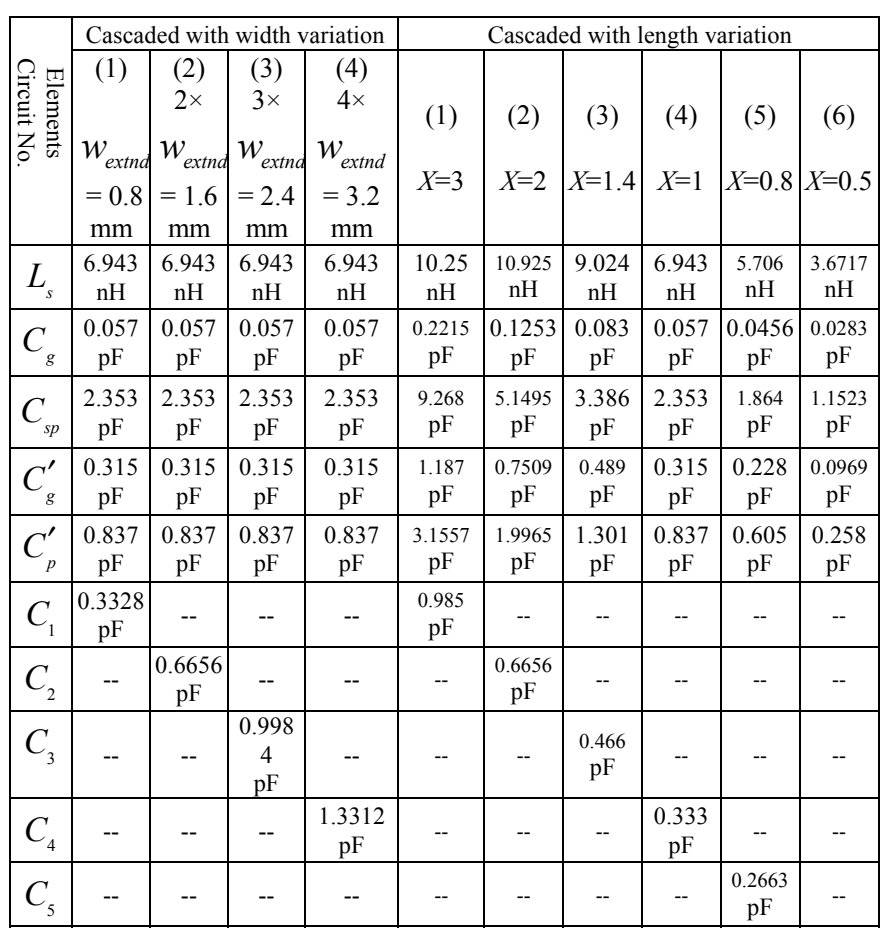

$X$ is the length multiplier compared to single modified stepped impedance resonator (shown in Fig-3).

In Fig- 8 the length of the cascaded modified stepped impedance resonator is varied along the $\mathrm{x}$-axis. For different length, cut-off frequency varies from minimum (leftmost lowpass filter) to higher (rightmost). As the area of lowpass 
filter decreases cut-off frequency increases. Again, observing the frequency response (fig-5) of the low-pass filter (fig-3), the stopband bandwidth is limited by harmonics, especially for the second harmonic. In order to extend the stop bandwidth, additional attenuation poles at the second harmonic can be added. The additional attenuation poles can be implemented by cascading small size lowpass modified stepped impedance filter. Thus we have a better broad stop band, - 60dB from $502 \mathrm{MHz}$ to $10 \mathrm{GHz}$.

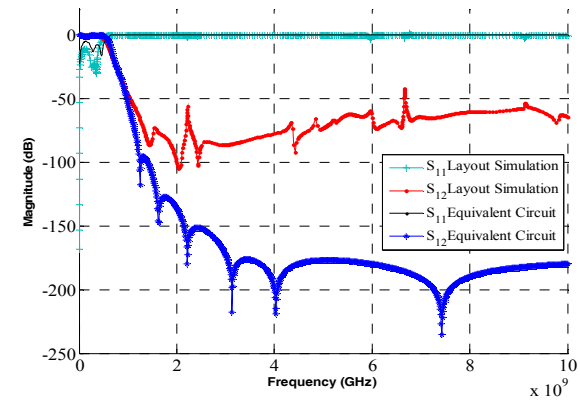

Fig. 9. Simulated results of the broad stopband lowpass filter with approximated dimensions while different length is considered.

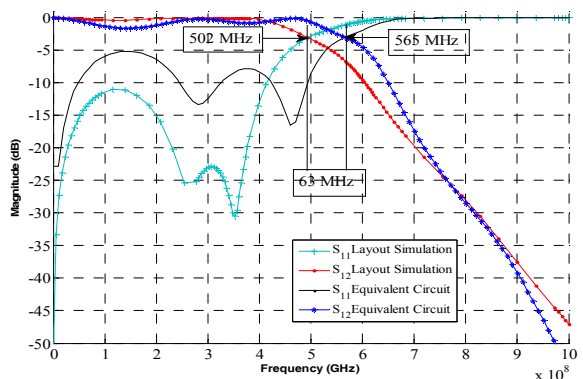

Fig. 10. Comparison of circuit model and EM simulation with approximation of dimension. Here, difference between these two cutoff frequencies is $63 \mathrm{MHz}$, return loss is lower than $-10 \mathrm{~dB}$ and passband ripple is less than $\pm 0.4 \mathrm{~dB}$

In Fig-9 and Fig-10, we have two results, one from EM simulation and other, from circuit model. Cutoff frequencies of these models differ from $63 \mathrm{MHz}$ which is because of negligence of inductance of series line and couple line. Insertion loss inside passband is less than $0.8 \mathrm{~dB}$, return loss is greater than $-12 \mathrm{~dB}$ for EM simulation and $-5 \mathrm{~dB}$ return loss for circuit simulation. The ripple inside the passband is $\pm 0.42 \mathrm{~dB}$

\section{CONCLUSION}

The proposed filter shows very good response as a stop band filter. The equivalent circuit proposed using transmission line theory and lumped elements shows very close result to EM simulation for the cut-off frequency, rejection and passband ripple. By varying the width and the length of the hairpin, the cutoff frequency of the filter can be modified. To obtain broad stop band and sharp rejection, the proposed structure can be cascaded. In both case, insertion loss is within the limit of operation. This broad stopband, compact, sharp rejection elliptical filter will be useful in many wireless communication systems.

\section{REFERENCES}

[1] M. Makimoto and S. Yamashita, "Bandpass filters using parallel coupled striplines stepped impedance resonators," IEEE Trans. Microw. Theory Tech, 1980, 12, (28) pp. 1423-1417.

[2] C. W. Tang, and S. F. You, "Miniaturized wide stopband rejected microstrip filter with coupled spur-lines," Electron. Lett, 2006, 42, (5) pp. 286-288.

[3] Y. Z. Wang and M. L. Her, "Compact microstrip bandpass filters using stepped-impedance resonator (SIR) and spur line sections," IEE Proc. Microw. Antennas Propag, 2006, 153,(5), pp. 435-440.

[4] K. X. Ma, J. G. Ma, K. S. Yeo and M. A. Do, "A compact size coupling controllable filter with separate electric and magnetic coupling paths," EEE Trans. Microw. Theory Tech, 2006, 54, (3), pp. 37-39.

[5] L. H. Hsieh and K. Chang, "Compact lowpass filter using stepped impedance hairpin resonator," "Electron. Let," Vol. 37, No. 14, pp. 899-900, July 2001.

[6] L. H. Hsieh and K. Chang, "Compact elliptical function lowpass filters using microstrip stepped impedance hairpin resonators." IEEE Trans. Microwave Theory Tech, Vol. 51, No. 1. Pp. 193-199, Jan 2003.

[7] L. H Hsieh and K. Chang, "Compact, broad-stopband elliptical-function lowpass filters using microstrip stepped impedance hairpin resonators," IEEE MTT -S, pp. 1775-1778, March 2003.

[8] M. H. Yang and J. Xu, "Design of compact, broad-stopband lowpass filter using modified stepped impedance hairpin resonators," Electon. Lett, Vol. 44 No. 20, September 2008.

[9] G. I. Zysman, A. K. Johnson, "Coupled Transmission line networks in an inhomogeneous dielectric medium" IEEE Trans. Microwave Theory Tech, Vol. 17, No. 10, October 1969.

[10] B. C. Wadell, "Transmission line Design Handbook," Norwood: “Artech House”, 1991, pp. 332. 